Bull. Austral. Math. Soc.

$65 \mathrm{D} 32,41 \mathrm{~A} 05,42 \mathrm{c} 05$

VOL. 57 (1998) [275-288]

\title{
CONVERGENCE OF A QUADRATURE FORMULA FOR VARIABLE-SIGNED WEIGHT FUNCTIONS
}

\author{
H.S. JUNG AND K.H. KWON
}

A quadrature formula for a variable-signed weight function $w(x)$ is constructed using Hermite interpolating polynomials. We show its mean and quadratic mean convergence. We also discuss the rate of convergence in terms of the modulus of continuity for higher order derivatives with respect to the sup norm.

\section{INTRODUCTION}

We consider a weight function $w(x)$ which is defined on a bounded interval $[a, b]$ such that all moments

$$
\int_{a}^{b} x^{i} w(x) d x, \quad i=0,1, \ldots
$$

are finite and $\operatorname{supp}(w)$ has a positive measure.

When $w(x) \geqslant 0$ on $[a, b]$, the theory of the Gauss - Christoffel quadrature formula is well developed (see [4] for an excellent survey on the subject), using Lagrange interpolation polynomials. In particular, the convergence of the corresponding Lagrange interpolation polynomials has been studied by many authors $[1,3,6,7]$ in various $L^{p}$. spaces.

In this work, we shall consider a variable-signed weight function $w(x)$. That is, we allow $w(x)$ to change its sign finitely many times in $(a, b)$. There are also many works on quadrature formulas for variable-signed weight functions (see [8] and [4, Chapter 3$]$ ).

We introduce a quadrature formula for variable signed weights, using Hermite interpolation polynomials.

For this quadrature formula, we shall prove mean and quadratic mean convergence of the corresponding Hermite interpolation polynomials in Section 3 and give a speed of rate of convergence for derivatives with respect to the sup norm in Section 4. Finally, we give some numerical examples in Section 5.

We refer to [9] and the references therein for weighted $L^{p}$ - convergence of Hermite interpolation polynomials based on the zeros of Jacobi polynomials.

Received 5th August, 1997

This work partially supported by KOSEF (95-0701-02-01-3), GARC and Korea Ministry of Education (BSRI 97-1420). The authors thank Professor Y. Xu for reading the manuscript and giving valuable comments.

Copyright Clearance Centre, Inc. Serial-fee code: 0004-9729/98 \$A2.00+0.00. 


\section{QUADRATURE FORMULA}

For any given $m(m \geqslant 1)$ distinct points $-\infty<a<\xi_{1}<\xi_{2}<\cdots<\xi_{m}<b<\infty$ and any $m$ nonnegative integers $\left\{\nu_{i}\right\}_{i=1}^{m}$, we let

$$
\phi(x)=\prod_{i=1}^{m}\left(x-\xi_{i}\right)^{\nu_{i}+1} \text { and } \nu:=\max \left\{\nu_{i} \mid 1 \leqslant i \leqslant m\right\} .
$$

For any $f \in C^{\nu}[a, b]$, we let $H(\phi ; f ; x)$ be the Hermite interpolation polynomial of $f$ relative to $\phi$. That is, $H(\phi ; f ; x)$ is the unique polynomial of degree $\leqslant \operatorname{deg}(\phi)-1$ satisfying

$$
H^{(j)}\left(\phi ; f ; x_{i}\right)=f^{(j)}\left(x_{i}\right), 1 \leqslant i \leqslant m \text { and } 0 \leqslant j \leqslant \nu_{i} .
$$

In fact, it is easy to see that

$$
H(\phi ; f ; x)=\sum_{i=1}^{m}\left[\sum_{j=0}^{\nu_{i}} \frac{1}{j !}\left(\frac{f(x)}{\phi_{i}(x)}\right)^{(j)}\left(\xi_{i}\right)\left(x-\xi_{i}\right)^{j}\right] \phi_{i}(x)
$$

where

$$
\phi_{i}(x)=\phi(x)\left(x-\xi_{i}\right)^{-\nu_{i}-1}, 1 \leqslant i \leqslant m .
$$

From now on, we assume that $w(x)$ is a weight function as in Section 1 for which we further assume that $w(x)$ changes its sign at $m$ distint points $\left\{\xi_{i}\right\}_{i=1}^{m}$ in $(a, b)$.

We let

$$
s(x):=\prod_{i=1}^{m}\left(x-\xi_{i}\right) \text { and } w^{*}(x):=w(x) s(x) .
$$

Then, we may assume that $w^{*}(x) \geqslant 0$ on $[a, b]$ and there is a unique monic orthogonal polynomial system $\left\{P_{n}\left(w^{*} ; x\right)\right\}_{n=0}^{\infty}=\left\{P_{n}(x)\right\}_{n=0}^{\infty}$ relative to the positive measure $w^{*}(x) d x$ on $[a, b]($ see $[3])$ :

$$
\int_{a}^{b} P_{m}(x) P_{n}(x) w^{*}(x) d x=K_{n} \delta_{m n}, m \text { and } n \geqslant 0
$$

where $K_{n}>0$. Moreover, $P_{n}(x), n \geqslant 1$, has $n$ real and simple zeros in $(a, b)$.

For any function $f(x) \in C^{1}[a, b]$ and any integer $n \geqslant 1$, we let

$$
H_{n}(f ; x):=H\left(P_{n} s ; f ; x\right)
$$

be the Hermite interpolation polynomial of $f$ relative to $P_{n} s$. Then, $H_{n}(f ; x)$ is a polynomial of degree $\leqslant n+m-1$ and

$$
H_{n}^{(j)}(f ; a)=f^{(j)}(a)
$$


for any zero $a$ of $P_{n}(x) s(x)$, where $j=0$ if $a$ is a simple zero of $P_{n}(x) s(x)$ and $j=0,1$ if $a$ is a double zero of $P_{n}(x) s(x)$. Moreover,

$$
H_{n}(\pi ; x)=\pi(x)
$$

for any polynomial $\pi(x)$ of degree $\leqslant n+m-1$.

For any fixed integer $n \geqslant 1$, we decompose $P_{n}(x) s(x)$ into

$$
P_{n}(x) s(x)=\prod_{1}^{l}\left(x-c_{i}\right)^{2} \prod_{1}^{m-l}\left(x-s_{i}\right) \prod_{1}^{n-l}\left(x-\eta_{i}\right)
$$

where $\left\{c_{i}\right\}_{i=1}^{l}$ are common zeros of $P_{n}(x)$ and $s(x)$ and $\left\{s_{i}\right\}_{i=1}^{m-l}$ and $\left\{\eta_{i}\right\}_{i=1}^{n-l}$ are zeros of only $s(x)$ and $P_{n}(x)$ respectively. Note that $l$ may depend on $n$. Then, we have from (2.1)

$$
\begin{aligned}
H_{n}(f ; x)= & \sum_{i=1}^{l}\left[\frac{f\left(c_{i}\right)}{P_{n}^{\prime}\left(c_{i}\right) s^{\prime}\left(c_{i}\right)}+\left(\frac{f(x)}{\phi_{i}^{c}(x)}\right)^{\prime}\left(c_{i}\right)\left(x-c_{i}\right)\right] \phi_{i}^{c}(x) \\
& \quad+\sum_{i=1}^{m-l} \frac{f\left(s_{i}\right)}{P_{n}\left(s_{i}\right) s^{\prime}\left(s_{i}\right)} \phi_{i}^{s}(x)+\sum_{i=1}^{n-l} \frac{f\left(\eta_{i}\right)}{P_{n}^{\prime}\left(\eta_{i}\right) s\left(\eta_{i}\right)} \phi_{i}^{\eta}(x),
\end{aligned}
$$

where

$$
\begin{aligned}
\phi_{i}^{c}(x) & =\frac{P_{n}(x) s(x)}{\left(x-c_{i}\right)^{2}}, 1 \leqslant i \leqslant l, \\
\phi_{i}^{s}(x) & =\frac{P_{n}(x) s(x)}{x-s_{i}}, 1 \leqslant i \leqslant m-l, \\
\phi_{i}^{\eta}(x) & =\frac{P_{n}(x) s(x)}{x-\eta_{i}}, 1 \leqslant i \leqslant n-l .
\end{aligned}
$$

In particular, if $f\left(c_{i}\right)=f^{\prime}\left(c_{i}\right)=0,1 \leqslant i \leqslant l$ and $f\left(s_{i}\right)=0,1 \leqslant i \leqslant m-l$, then

$$
H_{n}(f ; x)=\sum_{i=1}^{n-l} \frac{f\left(\eta_{i}\right)}{s\left(\eta_{i}\right)} \frac{P_{n}(x) s(x)}{P_{n}^{\prime}\left(\eta_{i}\right)\left(x-\eta_{i}\right)}
$$

We now let

$$
Q_{n}(w ; f):=\int_{a}^{b} H_{n}(f ; x) w(x) d x
$$

for any. $f(x) \in C^{1}[a, b]$ and any integer $n \geqslant 1$.

Since we have for any polynomial $\pi(x)$

$$
\pi(x)-H_{n}(\pi ; x)=P_{n}(x) s(x) Q(x),
$$


where $\operatorname{deg}(Q) \leqslant \operatorname{deg}(\pi)-n-m$,

$$
\int_{a}^{b} \pi(x) w(x) d x-Q_{n}(w ; \pi)=\int_{a}^{b} P_{n}(x) Q(x) w^{*}(x) d x .
$$

Since the right hand side of $(2.6)$ vanishes when $\operatorname{deg}(Q)<n$,

$$
\int_{a}^{b} \pi(x) w(x) d x=Q(w ; \pi)
$$

for any polynomial $\pi(x)$ of degree $\leqslant 2 n+m-1$. That is, the quadrature formula (2.5) has degree of exactness $2 n+m-1$ and

$$
\int_{a}^{b} \pi(x) w(x) d x=\lim _{n \rightarrow \infty} Q_{n}(w ; \pi)
$$

for any polynomial $\pi(x)$.

In the following sections, we discuss convergence of the quadrature formula (2.5) for arbitrary differentiable functions. The quadrature formula $Q_{n}(w ; f)$ in $(2.5)$ is essentially the same as the one in Struble [8], which he expressed it by using the partial fraction decomposition of

$$
\frac{1}{P_{n}(x) s(x)} \int_{a}^{b} \frac{P_{n}(z) s(z)-P_{n}(x) s(x)}{z-x} w(x) d x
$$

(see Theorem 1 and Theorem 3 in part II, [8]).

For later use, we let

$$
\begin{cases}l_{n}\left(w^{*} ; c_{i} ; x\right):=\frac{P_{n}(x)}{P_{n}^{\prime}\left(c_{i}\right)\left(x-c_{i}\right)}, & 1 \leqslant i \leqslant l \\ l_{n}\left(w^{*} ; \eta_{i} ; x\right):=\frac{P_{n}(x)}{P_{n}^{\prime}\left(\eta_{i}\right)\left(x-\eta_{i}\right)}, & 1 \leqslant i \leqslant n-l\end{cases}
$$

be the fundamental Lagrange polynomials at the nodes $\left\{c_{i}\right\}_{i=1}^{l}$ and $\left\{\eta_{i}\right\}_{i=1}^{n-l}$, which are zeros of the orthogonal polynomials $P_{n}(x)$. We also let

$$
\left\{\begin{array}{l}
\lambda_{n}\left(w^{*} ; c_{i}\right):=\int_{a}^{b} l_{n}\left(w^{*} ; c_{i} ; x\right) w^{*}(x) d x, \quad 1 \leqslant i \leqslant l, \\
\lambda_{n}\left(w^{*} ; \eta_{i}\right):=\int_{a}^{b} l_{n}\left(w^{*} ; \eta_{i} ; x\right) w^{*}(x) d x, \quad 1 \leqslant i \leqslant n-l
\end{array}\right.
$$

be the corresponding Christoffel numbers. Then (see [3])

$$
\lambda_{n}\left(w^{*} ; c_{i}\right)>0,1 \leqslant i \leqslant l \text { and } \lambda_{n}\left(w^{*} ; \eta_{i}\right)>0,1 \leqslant i \leqslant n-l
$$

and

$$
\sum_{i=1}^{l} \lambda_{n}\left(w^{*} ; c_{i}\right)+\sum_{i=1}^{n-l} \lambda_{n}\left(w^{*} ; \eta_{i}\right)=\int_{a}^{b} w^{*}(x) d x
$$




\section{Convergence in mean}

We now discuss mean and quadratic mean convergence of the quadrature formula (2.5). We shall use the following, which generalises the well known Bernstein theorem (see [2]): For any integer $k \geqslant 0$ and any function $f \in C^{k}[0,1]$, the Bernstein polynomials

$$
B_{n}(f ; x):=\sum_{j=0}^{n} f\left(\frac{j}{n}\right)\left(\begin{array}{l}
n \\
j
\end{array}\right) x^{j}(1-x)^{n-j}, \quad n \geqslant 1
$$

satisfy

$$
\lim _{n \rightarrow \infty}\left\|B_{n}^{(j)}(f ; x)-f^{(j)}(x)\right\|=0, \quad 0 \leqslant j \leqslant k,
$$

where $\|\cdot\|$ denotes the sup norm on $[0,1]$.

Proposition 3.1. Let $s(x)$ be as in Section 1 and let $a=0, b=1$. Then for any $f \in C^{k}[0,1](k \geqslant 0$ an integer) and any $\varepsilon>0$, there is a polynomial $\pi(x)$ such that

$$
\pi^{(r)}\left(\xi_{i}\right)=f^{(r)}\left(\xi_{i}\right), 1 \leqslant i \leqslant m \text { and } 0 \leqslant r \leqslant k
$$

and

$$
\left|\pi^{(r)}(x)-f^{(r)}(x)\right| \leqslant|s(x)|^{k-r} \varepsilon, 0 \leqslant r \leqslant k \text { and } 0 \leqslant x \leqslant 1 .
$$

In particular, $f^{(r)}(x), 0 \leqslant r \leqslant k$, can be uniformly approximated on $[0,1]$ by polynomials satisfying (3.1).

Proof: For any integer $n \geqslant 1$, we let

$$
\Psi_{n}(f ; x):=B_{n}(f ; x)+H\left(s^{k+1} ; f-B_{n}(f ; x) ; x\right) .
$$

Then, $\operatorname{deg} \Psi_{n}(f ; x) \leqslant \max (n,(k+1) m-1)$ and

$$
\Psi_{n}^{(j)}\left(f ; \xi_{i}\right)=f^{(j)}\left(\xi_{i}\right), 1 \leqslant i \leqslant m \text { and } 0 \leqslant j \leqslant k .
$$

That is, $\Psi_{n}(f ; x)$ satisfies condition $(3.1)$ for any $n \geqslant 1$. We now show that $\Psi_{n}(f ; x)$ satisfies condition (3.2) for large enough $n$.

We may express the Hermite interpolating polynomial $H\left(s^{k+1} ; f ; x\right)$ as

$$
H\left(s^{k+1} ; f ; x\right)=\sum_{j=0}^{k} \sum_{i=1}^{m} f^{(j)}\left(\xi_{i}\right) h_{i j}(x)
$$

where $h_{i j}(x)$ is the unique polynomial of degree $\leqslant(k+1) m-1$ satisfying

$$
h_{i j}^{(q)}\left(\xi_{p}\right)=\delta_{i p} \delta_{j q}, 1 \leqslant i, p \leqslant m \text { and } 0 \leqslant j, q \leqslant k
$$


Then for any $r$ with $0 \leqslant r \leqslant k$

$$
\left|H^{(r)}\left(s^{k+1} ; f ; x\right)\right| \leqslant \sum_{j=0}^{k} \sum_{i=1}^{m}\left|f^{(j)}\left(\xi_{i}\right)\right|\left|h_{i j}^{(r)}(x)\right| \leqslant C_{r} \max _{0 \leqslant j \leqslant k}\left\|f^{(j)}\right\|,
$$

where $C_{r}:=\sum_{j=0}^{k} \sum_{i=1}^{m}\left\|h_{i j}^{(r)}\right\|$. Hence,

$$
\begin{aligned}
\left|f^{(r)}(x)-\Psi_{n}^{(r)}(f ; x)\right| & \leqslant\left|f^{(r)}(x)-B_{n}^{(r)}(f ; x)\right|+\left|H^{(r)}\left(s^{k+1} ; f-B_{n}(f ; x) ; x\right)\right| \\
& \leqslant\left(1+C_{r}\right) \max _{0 \leqslant j \leqslant k}\left\|f^{(j)}-B_{n}^{(j)}(f ; x)\right\|, 0 \leqslant x \leqslant 1
\end{aligned}
$$

so that

$$
\lim _{n \rightarrow \infty}\left\|f^{(r)}-\Psi_{n}^{(r)}(f ; x)\right\|=0, \quad 0 \leqslant r \leqslant k
$$

by Bernstein's theorem. We now consider the inequality $(3.2)$ for $\pi(x)=\Psi_{n}(f ; x)$ with large $n$.

If $x=\xi_{i}, 1 \leqslant i \leqslant m$, then the left hand side of inequality (3.2) is equal to 0 . Choose $\delta>0$ such that $0<\delta<\min \left\{\left(\xi_{i+1}-\underset{m}{\xi_{i}}\right) / 2 \mid 0 \leqslant i \leqslant m\right\}$, where $\xi_{0}=0$ and $\xi_{m+1}=1$. Let $A_{i}=\left[\xi_{i}-\delta, \xi_{i}+\delta\right], 1 \leqslant i \leqslant m, A=\bigcup_{1}^{m} A_{i}$, and $B=[0,1] \backslash \operatorname{Int}(A)(\operatorname{Int}(A)$ denotes the interior of $A$ ). Then $A_{i} \cap\left\{\xi_{j}\right\}_{j=1}^{m}=\left\{\xi_{i}\right\}, 1 \leqslant i \leqslant m$, and $B \cap\left\{\xi_{j}\right\}_{j=1}^{m}=\emptyset$.

If $x \in A_{i} \backslash\left\{\xi_{i}\right\}$ for some $i=1,2, \cdots, m$, then we have, by using (3.1) and repeated applications of the mean value theorem,

$$
\begin{aligned}
\left|f^{(r)}(x)-\Psi_{n}^{(r)}(f ; x)\right| & =\left|f^{(k)}(\tilde{x})-\Psi_{n}^{(k)}(f ; \tilde{x})\right|\left|x-\xi_{i}\right|^{k-r} \\
& \leqslant M_{A}\left\|f^{(k)}-\Psi_{n}^{(k)}(f ; x)\right\||s(x)|^{k-r}
\end{aligned}
$$

where $\widetilde{x}$ is some point in $A_{i}$ and $M_{A}:=\max _{1 \leqslant i \leqslant m} \max _{x \in A_{i}}\left\{\left|\left(x-\xi_{i}\right) /(s(x))\right|^{k-r}\right\}$.

If $x \in B$, then

$$
\left|f^{(r)}(x)-\Psi_{n}^{(r)}(f ; x)\right| \leqslant M_{B}\left\|f^{(r)}-\Psi_{n}^{(r)}(f ; x)\right\||s(x)|^{k-r}
$$

where $M_{B}:=\max \left\{|s(x)|^{r-k} \mid x \in B\right\}$.

Therefore, we have the inequality (3.2) for $\pi(x)=\Psi_{n}(f ; x)$ if $n$ is large enough by (3.4), (3.5), and (3.6).

Note that while Bernstein polynomials $B_{n}(f ; x)$ approximate $f(x)$ uniformly on $[0,1]$ with $f(0)$ and $f(1)$ fixed, polynomials $\Psi_{n}(f ; x)$ approximate $f(x)$ uniformly on $[0,1]$ with $f^{(j)}\left(\xi_{i}\right), 1 \leqslant i \leqslant m$ and $0 \leqslant j \leqslant k$, fixed. When $m=0$, Proposition 3.1 becomes Bernstein's theorem by taking $s(x) \equiv 1$.

We are now ready to prove mean and quadratic mean convergence of the quadrature formula (2.5). 
THEOREM 3.2. (Mean convergence) For any $f \in C^{1}[a, b]$,

$$
\lim _{n \rightarrow \infty} Q_{n}(w ; f)=\int_{a}^{b} f(x) w(x) d x
$$

Proof: We may assume that $a=0$ and $b=1$. Then for any polynomial $\pi(x)$ satisfying (3.1) with $k=1$, we have by (2.4) and (2.7)

$$
H_{n}(\pi-f ; x)=\sum_{i=1}^{n-l} \frac{\pi\left(\eta_{i}\right)-f\left(\eta_{i}\right)}{s\left(\eta_{i}\right)} \frac{P_{n}(x) s(x)}{P_{n}^{\prime}\left(\eta_{i}\right)\left(x-\eta_{i}\right)}=\sum_{i=1}^{n-l} \frac{\pi\left(\eta_{i}\right)-f\left(\eta_{i}\right)}{s\left(\eta_{i}\right)} s(x) l_{n}\left(w^{*} ; \eta_{i} ; x\right)
$$

so that (see (2.8))

$$
Q_{n}(w ; \pi-f)=\int_{0}^{1} H_{n}(\pi-f ; x) w(x) d x=\sum_{i=1}^{n-l} \frac{\pi\left(\eta_{i}\right)-f\left(\eta_{i}\right)}{s\left(\eta_{i}\right)} \lambda_{n}\left(w^{*} ; \eta_{i}\right)
$$

Hence

$$
\int_{0}^{1} f(x) w(x) d x-Q_{n}(w ; f)=\int_{0}^{1}[f(x)-\pi(x)] w(x) d x+Q_{n}(w ; \pi-f)
$$

provided that $\operatorname{deg}(\pi) \leqslant n+m-1$ and so (see 3.7 )

$$
\begin{aligned}
\left|\int_{0}^{1} f(x) w(x) d x-Q_{n}(w ; f)\right| \leqslant & \int_{0}^{1}|f(x)-\pi(x)||w(x)| d x \\
& +\sum_{i=1}^{n-l}\left|\frac{\pi\left(\eta_{i}\right)-f\left(\eta_{i}\right)}{s\left(\eta_{i}\right)}\right| \lambda_{n}\left(w^{*} ; \eta_{i}\right) .
\end{aligned}
$$

For any $\varepsilon>0$, we may take by Proposition 3.1

$$
\pi(x)=\Psi_{p}(f ; x)=B_{p}(f ; x)+H\left(s^{2} ; f-B_{p}(f ; x) ; x\right)
$$

where $p$ is so large that

$$
\left|f^{(r)}(x)-\pi^{(r)}(x)\right| \leqslant|s(x)|^{1-r} \varepsilon, r=0,1 \text { and } 0 \leqslant x \leqslant 1 .
$$

Then

$$
\begin{aligned}
\left|\int_{0}^{1} f(x) w(x) d x-Q_{n}(w ; f)\right| \leqslant & \int_{0}^{1}|f(x)-\pi(x)||w(x)| d x \\
& +\sum_{i=1}^{n-l}\left|\frac{\pi\left(\eta_{i}\right)-f\left(\eta_{i}\right)}{s\left(\eta_{i}\right)}\right| \lambda_{n}\left(w^{*} ; \eta_{i}\right) \\
\leqslant & \varepsilon \int_{0}^{1} w^{*}(x) d x+\varepsilon \sum_{i=1}^{n-l} \lambda_{n}\left(w^{*} ; \eta_{i}\right) \\
\leqslant & 2 \varepsilon \int_{0}^{1} w^{*}(x) d x
\end{aligned}
$$

(see (2.9)) if $n$ is so large that $\operatorname{deg}(\pi)=\operatorname{deg}\left(\Psi_{p}(f ; x)\right) \leqslant n+m-1$. Since $\varepsilon>0$ is arbitrary, the conclusion of the theorem follows. 
THEOREM 3.3. (Quadratic mean convergence) For any $f \in C^{1}[a, b]$

$$
\lim _{n \rightarrow \infty} \int_{a}^{b}\left[f(x)-H_{n}(f ; x)\right]^{2}|w(x)| d x=0 .
$$

PRoof: We may assume that $a=0$ and $b=1$. Then for any polynomial $\pi(x)$ of degree $\leqslant n+m-1$, we have

$$
\begin{aligned}
\int_{0}^{1}[f(x) & \left.-H_{n}(f ; x)\right]^{2}|w(x)| d x=\int_{0}^{1}[f(x)-\pi(x)]^{2}|w(x)| d x \\
& +\int_{0}^{1} H_{n}^{2}(\pi-f ; x)|w(x)| d x+2 \int_{0}^{1}[f(x)-\pi(x)] H_{n}(\pi-f ; x)|w(x)| d x
\end{aligned}
$$

so that

$$
\begin{aligned}
& \int_{0}^{1}\left[f(x)-H_{n}(f ; x)\right]^{2}|w(x)| d x \\
& \quad \leqslant 2\left(\int_{0}^{1}[f(x)-\pi(x)]^{2}|w(x)| d x+\int_{0}^{1} H_{n}^{2}(\pi-f ; x)|w(x)| d x\right) .
\end{aligned}
$$

Now, for any $\varepsilon>0$, choose $\pi(x)$ as

$$
\pi(x)=\Psi_{p}(f ; x)=B_{p}(f ; x)+H\left(s^{2} ; f-B_{p}(f ; x) ; x\right)
$$

so that (3.1) and (3.2) hold with $k=1$. Then

$$
\int_{0}^{1}[f(x)-\pi(x)]^{2}|w(x)| d x \leqslant \varepsilon^{2} \int_{0}^{1}|s(x)|^{2}|w(x)| d x \leqslant \varepsilon^{2}\|s\| \int_{0}^{1} w^{*}(x) d x
$$

(here $\|s\|=\max _{0 \leqslant x \leqslant 1}|s(x)|$ ) and

$$
\begin{aligned}
\int_{0}^{1} H_{n}^{2}(\pi-f ; x)|w(x)| d x & =\int_{0}^{1}\left(\sum_{i=1}^{n-l} \frac{\pi\left(\eta_{i}\right)-f\left(\eta_{i}\right)}{s\left(\eta_{i}\right)} l_{n}\left(w^{*} ; \eta_{i} ; x\right)\right)^{2} s^{2}(x)|w(x)| d x \\
& =\|s\| \int_{0}^{1}\left(\sum_{i=1}^{n-l} \frac{\pi\left(\eta_{i}\right)-f\left(\eta_{i}\right)}{s\left(\eta_{i}\right)} l_{n}\left(w^{*} ; \eta_{i} ; x\right)\right)^{2} w^{*}(x) d x \\
& =\|s\| \sum_{i=1}^{n-l}\left(\frac{\pi\left(\eta_{i}\right)-f\left(\eta_{i}\right)}{s\left(\eta_{i}\right)}\right)^{2} \lambda_{n}\left(w^{*} ; \eta_{i}\right)
\end{aligned}
$$

since $\int_{0}^{1} l_{n}\left(w^{*} ; \eta_{i} ; x\right) l_{n}\left(w^{*} ; \eta_{j} ; x\right) w^{*}(x) d x=\lambda_{n}\left(w^{*} ; \eta_{i}\right) \delta_{i j}$ (see $\left.[\mathbf{3}]\right)$. Hence

$$
\begin{aligned}
\int_{0}^{1} H_{n}^{2}(\pi-f ; x)|w(x)| d x & \leqslant \varepsilon^{2}\|s\| \sum_{i=1}^{n-l} \lambda_{n}\left(w^{*} ; \eta_{i}\right) \\
& \leqslant \varepsilon^{2}\|s\| \int_{0}^{1} w^{*}(x) d x
\end{aligned}
$$


(see (2.9)) if $n$ is so large that $\operatorname{deg}(\pi)=\operatorname{deg}\left(\Psi_{p}(f ; x)\right) \leqslant n+m-1$. Therefore, we have

$$
\int_{0}^{1}\left[f(x)-H_{n}(f ; x)\right]^{2}|w(x)| d x \leqslant 4 \varepsilon^{2}\|s\| \int_{0}^{1} w^{*}(x) d x .
$$

Since $\varepsilon>0$ is arbitrary, the conclusion of the theorem follows.

\section{Convergence in SUP NORM}

Now, we investigate the convergence speed of derivatives of the Hermite interpolation polynomials $H_{n}(f ; x)$ with respect to the sup norm. For the sake of convenience, we assume $a=-1$ and $b=1$ and use

$$
\|f\|:=\sup _{[-1,1]}|f(x)|
$$

We need the following two facts.

LEMMA 4.1. (Markov's inequality) For any polynomial $\pi_{n}(x)$ of degree $\leqslant n$ and any integer $r \geqslant 0$

$$
\left\|\pi_{n}^{(r)}(x)\right\|=O(1) n^{2 r}\left\|\pi_{n}\right\|
$$

LEMMA 4.2. (Gopengauz's theorem [5]) Let $f \in C^{k}[-1,1], k \geqslant 0$ an integer. Then for any integer $n \geqslant 4 k+5$, there is a polynomial $G_{n}(x)$ of degree $\leqslant n$ such that

$$
\left|f^{(r)}(x)-G_{n}^{(r)}(x)\right|=O(1) n^{r-k}\left(1-x^{2}\right)^{(k-r) / 2} \omega\left(f^{(k)} ; \frac{1}{n}\right), \quad 0 \leqslant r \leqslant k \text { and }-1 \leqslant x \leqslant 1,
$$

where $\omega(g ; h):=\sup _{|x-y| \leqslant h}|g(x)-g(y)|$ is the modulus of continuity for $g$.

The following theorem is motivated by results of Balazs and Kilgore [1].

TheOREM 4.3. Let $f \in C^{k}[-1,1], k \geqslant 0$ an integer. Then for any integer $n \geqslant \max \{4 k+5,2 m-1\}$,

$$
\left\|H_{n}^{(r)}(f ; x)-f^{(r)}(x)\right\|=O(1) n^{2 r+1-k} \omega\left(f^{(k)} ; \frac{1}{n}\right) L_{n}
$$

where $L_{n}:=\left\|\sum_{i=1}^{l}\left|l_{n i}\left(w^{*} ; c_{i} ; x\right)\right|+\sum_{i=1}^{n-l}\left|l_{n i}\left(w^{*} ; \eta_{i} ; x\right)\right|\right\|$ is the Lebesgue constant for the positive weight function $w^{*}(x)$.

Proof: Assume $n \geqslant \max \{4 k+5,2 m-1\}$ and let

$$
R_{n}(x):=G_{n}(x)+H\left(s^{2} ; f-G_{n} ; x\right)
$$


where $G_{n}(x)$ is a polynomial as in Gopengauz's theorem. Then $\operatorname{deg}\left(R_{n}\right) \leqslant n$ so that

$$
H_{n}\left(R_{n} ; x\right)=R_{n}(x) \quad \text { and } \quad R_{n}^{(j)}\left(\xi_{i}\right)=f^{(j)}\left(\xi_{i}\right), \quad 1 \leqslant i \leqslant m \quad \text { and } \quad j=0,1
$$

and

$$
\left\|H_{n}^{(r)}(f ; x)-f^{(r)}(x)\right\| \leqslant\left\|H_{n}^{(r)}\left(f-R_{n} ; x\right)\right\|+\left\|R_{n}^{(r)}-f^{(r)}\right\| \quad 0 \leqslant r \leqslant k .
$$

By (3.3) and (4.2), we have

$$
\begin{aligned}
&\left|R_{n}^{(r)}(x)-f^{(r)}(x)\right| \leqslant\left|G_{n}^{(r)}(x)-f^{(r)}(x)\right|+\left|H^{(r)}\left(s^{2} ; f-G_{n} ; x\right)\right| \\
& \leqslant O(1) n^{r-k} \omega\left(f^{(k)} ; \frac{1}{n}\right)+O(1) n^{-k} \omega\left(f^{(k)} ; \frac{1}{n}\right)+O(1) n^{1-k} \omega\left(f^{(k)} ; \frac{1}{n}\right) \\
&= \begin{cases}O(1) n^{1-k} \omega\left(f^{(k)} ; \frac{1}{n}\right) & \text { if } \quad r=0 \\
O(1) n^{r-k} \omega\left(f^{(k)} ; \frac{1}{n}\right) & \text { if } \quad 1 \leqslant r \leqslant k .\end{cases}
\end{aligned}
$$

On the other hand, since $R_{n}^{(j)}\left(\xi_{i}\right)=f^{(j)}\left(\xi_{i}\right), 1 \leqslant i \leqslant m$ and $j=0,1$, we have (see (2.4))

$$
H_{n}\left(f-R_{n} ; x\right)=\sum_{i=1}^{n-l} \frac{f\left(\eta_{i}\right)-R_{n}\left(\eta_{i}\right)}{s\left(\eta_{i}\right)} l_{n}\left(w^{*} ; \eta_{i} ; x\right) s(x)
$$

We can easily see that

$$
\left|f(x)-R_{n}(x)\right|=O(1) n^{1-k} \omega\left(f^{(k)} ; \frac{1}{n}\right)|s(x)|, \quad-1 \leqslant x \leqslant 1
$$

(the proof is the same as the one for Proposition 3.1: replace $\Psi_{n}(f ; x)$ by $R_{n}(x)$ and set $r=0, k=0$ in (3.5) and (3.6) and then apply (4.4)), so that

$$
\left\|\frac{f\left(\eta_{i}\right)-R_{n}\left(\eta_{i}\right)}{s\left(\eta_{i}\right)}\right\|=O(1) n^{1-k} \omega\left(f^{(k)} ; \frac{1}{n}\right), \quad 1 \leqslant i \leqslant n-l .
$$

Therefore, by Markov's inequality and (4.4), we have

$$
\begin{aligned}
\left|H_{n}^{(r)}\left(f-R_{n} ; x\right)\right| & =\left|\sum_{i=1}^{n-l} \frac{f\left(\eta_{i}\right)-R_{n}\left(\eta_{i}\right)}{s\left(\eta_{i}\right)}\left(l_{n}\left(w^{*} ; \eta_{i} ; x\right) s(x)\right)^{(r)}\right| \\
& =O(1)(n+m-1)^{2 r}\left\|\sum_{i=1}^{n-l} \frac{f\left(\eta_{i}\right)-R_{n}\left(\eta_{i}\right)}{s\left(\eta_{i}\right)} l_{n}\left(w^{*} ; \eta_{i} ; x\right) s(x)\right\| \\
& =O(1)\|s\|(n+m-1)^{2 r} n^{1-k} \omega\left(f^{(k)} ; \frac{1}{n}\right)\left\|\sum_{i=1}^{n-l}\left|l_{n}\left(w^{*} ; \eta_{i} ; x\right)\right|\right\| \\
& =O(1) n^{2 r+1-k} \omega\left(f^{(k)} ; \frac{1}{n}\right) L_{n} .
\end{aligned}
$$

Now, the conclusion follows from (4.3), (4.4), and (4.6) since $L_{n} \geqslant 1$. 


\section{NUMERICAL EXAMPLE}

In this section, we illustrate our quadrature formula (2.5) by estimating

$$
\int_{-1}^{1} f(x) \tan 1.5 x d x
$$

in two different ways. First, we view the integral (5.1) as an integral of $f(x)$ with respect to the sign changing weight function $\tan 1.5 x$ and approximate it by using the quadrature formula (2.5). Secondly, we view the integral (5.1) as an integral of $f(x) \tan 1.5 x$ with respect to the Legendre weight 1 on $[-1,1]$ and approximate it by the classical GaussLegendre quadrature formula (see [3]).

In the following, we let

$$
\begin{aligned}
w(x) & :=\tan 1.5 x, w^{*}(x):=x \tan 1.5 x, \\
L_{n}(x) & :=n-\operatorname{th} \text { Legendre polynomial, } \\
P_{n}(x) & :=n-\text { th orthogonal polynomial relative to } w^{*}(x) \text { on }[-1,1], \\
\left\{z_{n k}\right\}_{k=1}^{n} & :=\text { zeros of } L_{n}(x) \text { for } n \geqslant 1 .
\end{aligned}
$$

It is well known that Legendre polynomials $\left\{L_{n}(x)\right\}_{n=0}^{\infty}$ and $\left\{P_{n}(x)\right\}_{n=0}^{\infty}$ satisfy the three term recurrence relations:

$$
\begin{aligned}
(n+1) L_{n+1}(x) & =(2 n+1) x L_{n}(x)-n L_{n-1}(x), n \geqslant 0,\left(L_{-1} \equiv 0, L_{0}(x)=1\right) \\
P_{n+1}(x) & =x P_{n}(x)-C_{n} P_{n-1}(x), n \geqslant 0,\left(P_{-1} \equiv 0, P_{0}(x)=1\right)
\end{aligned}
$$

where

$$
C_{n}=\frac{\int_{-1}^{1} x P_{n}(x) P_{n-1}(x) w^{*}(x) d x}{\int_{-1}^{1} P_{n-1}^{2}(x) w^{*}(x) d x}=\frac{\int_{-1}^{1} P_{n}^{2}(x) w^{*}(x) d x}{\int_{-1}^{1} P_{n-1}^{2}(x) w^{*}(x) d x}, n \geqslant 1 .
$$

We note that $P_{n}(x)$ is even or odd if $n$ is even or odd respectively since $w^{*}(x)$ is even.

The Gauss-Legendre quadrature formula is given by

$$
\begin{aligned}
Q L & :=Q_{n+1}(1 ; f w) \\
& =\sum_{k=1}^{n+1} \mu_{L, n+1, k} f\left(z_{n+1, k}\right) w\left(z_{n+1, k}\right)
\end{aligned}
$$

where $\mu_{L, n+1, k}$ are Christoffel numbers with respect to the Legendre weight 1 , that is,

$$
\mu_{L, n+1, k}=\int_{-1}^{1} \frac{L_{n+1}(x)}{\left(x-z_{n+1, k}\right) L_{n+1}^{\prime}\left(z_{n+1, k}\right)} d x .
$$

On the other hand, the quadrature formula with respect to $w(x)$ is given by

$$
Q S:=Q_{n}(w ; f)=\int_{-1}^{1} H_{n}(f ; x) w(x) d x
$$


If $n$ is even, then $P_{n}(x)$ is even and $l=0, m=1, s_{1}=0$, and $\left\{\eta_{i}\right\}_{i=1}^{n}$ are zeros of $P_{n}(x)$. Then we have from $(2.3)$

$$
H_{n}(f ; x)=\frac{f(0)}{P_{n}(0)} P_{n}(x)+\sum_{i=1}^{n} \frac{f\left(\eta_{i}\right)}{\eta_{i}\left(x-\eta_{i}\right) P_{n}^{\prime}\left(\eta_{i}\right)} P_{n}(x) x
$$

so that

$$
\begin{aligned}
Q_{n}(w ; f) & =\frac{f(0)}{P_{n}(0)} \int_{-1}^{1} P_{n}(x) w(x) d x+\sum_{i=1}^{n} \frac{\lambda_{n}\left(w^{*} ; \eta_{i}\right)}{\eta_{i}} f\left(\eta_{i}\right) \\
& =\sum_{i=1}^{n} \frac{\lambda_{n}\left(w^{*} ; \eta_{i}\right)}{\eta_{i}} f\left(\eta_{i}\right)=\sum_{i=1}^{n} \mu_{s, n, i} f\left(\eta_{i}\right)
\end{aligned}
$$

where $\mu_{s, n, i}=\lambda_{n}\left(w^{*} ; \eta_{i}\right) / \eta_{i}, i=1,2, \cdots, n$.

If $n$ is odd, then $P_{n}(x)$ is odd so that $P_{n}(0)=0$ and $l=m=1, c_{1}=0$, and $\left\{\eta_{i}\right\}_{i=1}^{n-1}$ are non-zero zeros of $P_{n}(x)$. Then we have from (2.3)

$$
H_{n}(f ; x)=\frac{f(0)}{x P_{n}^{\prime}(0)} P_{n}(x)+\frac{f^{\prime}(0)}{P_{n}^{\prime}(0)} P_{n}(x)+\sum_{i=1}^{n-1} \frac{f\left(\eta_{i}\right)}{\eta_{i} P_{n}^{\prime}\left(\eta_{i}\right)\left(x-\eta_{i}\right)} P_{n}(x) x
$$

so that

$$
\begin{aligned}
Q_{n}(w ; f) & =\frac{f^{\prime}(0)}{P_{n}^{\prime}(0)} \int_{-1}^{1} P_{n}(x) w(x) d x+\sum_{i=1}^{n-1} \frac{\lambda_{n}\left(w^{*} ; \eta_{i}\right)}{\eta_{i}} f\left(\eta_{i}\right) \\
& =\sum_{i=1}^{n-1} \mu_{s, n, i} f\left(\eta_{i}\right)+\mu_{s, n, n} f^{\prime}(0)
\end{aligned}
$$

where

$$
\mu_{s, n, i}:=\frac{\lambda_{n}\left(w^{*} ; \eta_{i}\right)}{\eta_{i}}, i=1,2, \cdots, n-1
$$

and

$$
\mu_{s, n, n}:=\frac{\int_{-1}^{1} P_{n}(x) w(x) d x}{P_{n}^{\prime}(0)} .
$$

We used Maple V Release 4 for the numeric computations below. In Table I, $\eta_{k}$, $z_{n+1, k}$ and $\mu_{s, n, k}, \mu_{L, n+1, k}$ are given for $n=9$. Zeros $\eta_{k}$ and $z_{n+1, k}$ for $P_{n}(x)$ and $L_{n+1}(x)$ are computed using the three term recurrence relations (5.2) and (5.3). For comparison, we set

$$
E S:=\left|\int_{-1}^{1} f(x) w(x) d x-Q S\right| ; \quad E L:=\left|\int_{-1}^{1} f(x) w(x) d x-Q L\right| .
$$

Table II gives numerical results of the comparison between $Q S$ and $Q L$ for several choices of function $f(x)$. Here, $I$ is rounded off in the true value of $\int_{-1}^{1} f(x) \tan 1.5 x d x$. 


\begin{tabular}{rrr|rr}
\hline & \multicolumn{2}{c|}{ Legendre weight } & \multicolumn{2}{c}{$w(x):=\tan (1.5 x)$} \\
\hline$k$ & $z_{10, k}$ & $\mu_{L, 10, k}$ & \multicolumn{1}{c}{$\eta_{k}$} & \multicolumn{1}{c}{$\mu_{s, 9, k}$} \\
\hline 1 & .97390653 & .06667135 & .98101119 & .51196269 \\
2 & .86506337 & .14945134 & .88715108 & .56855905 \\
3 & .67940957 & .21908636 & .70328452 & .39919132 \\
4 & .43339539 & .26926672 & .43963347 & .23197168 \\
5 & .14887434 & .29552422 & -.43963347 & -.23197168 \\
6 & -.14887434 & .29552422 & -.70328452 & -.39919132 \\
7 & -.43339539 & .26926672 & -.88715108 & -.56855905 \\
8 & -.67940957 & .21908636 & -.98101119 & -.51196269 \\
9 & -.86506337 & .14945134 & .00000000 & .01474570 \\
10 & -.97390653 & .06667135 & & \\
\hline
\end{tabular}

TABLE I $(n=9)$

\begin{tabular}{rrrrrr}
\hline$f(x)$ & $\mathrm{I}$ & $Q S$ & $Q L$ & $E S$ & $E L$ \\
\hline$x$ & 2.7934786 & 2.7934787 & 2.7804519 & .0000000 & .0130268 \\
$x^{5}$ & 1.7001891 & 1.7001891 & 1.6845234 & .0000000 & .0156657 \\
$x^{11}$ & 1.1505534 & 1.1505534 & 1.1298938 & .0000000 & .0206596 \\
$x^{15}$ & .9607962 & .9607962 & .9359513 & .0000000 & .0248448 \\
$x^{21}$ & .7771238 & .7770607 & .7443576 & .0000631 & .0327662 \\
$\sin (x)$ & 2.4610620 & 2.4610621 & 2.4502890 & .0000001 & .0107730 \\
$\tan (x)$ & 3.8356255 & 3.8356257 & 3.8140796 & .0000002 & .0215459 \\
$\sin (5 x)$ & -1.9418394 & -1.9418395 & -1.9310664 & .0000001 & .0107730 \\
$\sinh (5 x)$ & 132.6488405 & 132.6488421 & 131.4800797 & .0000016 & 1.1687608 \\
$e^{x}$ & 3.1542388 & 3.1542388 & 3.1386971 & .0000000 & .0155417 \\
$\frac{1}{1.2+x}$ & -4.4159041 & -4.4158277 & -4.3780213 & .0000763 & .0378828 \\
\hline
\end{tabular}

TABLE II $(n=9)$

\section{REFERENCES}

[1] K. Balazs and T. Kilgore, 'On the simultaneous approximation of derivatives by Lagrange and Hermite interpolation', J. Approx. Theory 60 (1990), 231-244.

[2] P.J. Davis, Interpolation and approximation (Blaisdell, New York, 1963).

[3] G. Freud, Orthogonal polynomials (Pergamon Press, Oxford, 1971).

[4] W. Gautschi, 'A survey of Gauss - Christoffel quadrature formula', in E.B. Christoffel Symp., (P.L. Butzer and F. Fehér, Editors) (Birkhäuser, Basel, 1981), pp. 72-147.

[5] I.E. Gopengauz, 'On a theorem of A.F. Timan on approximation of function by polynomials on a finite interval', (in Russian) 1967, Mat. Zametki 1 (), 163-172; (English translation, Math. Notes I (1967), 110-116).

[6] G. Mastroianni and P. Nevai, 'Mean convergence of derivatives of Lagrange interpolation', J. Comput. Appl. Math. 34 (1991), 385-396. 
[7] P. Nevai, 'Mean convergence of Lagrange interpolation, III', Trans. Amer. Math. Soc. 282 (1984), 669-698.

[8] G.W. Struble, Orthogonal polynomials: Variable - signed weight functions, Ph.D. Thesis (University of Wisconsin, 1961).

[9] P. Vértesi and Y. Xu, 'Truncated Hermite interpolation polynomials', Studia Sci. Math. Hungar. 23 (1993), 205-213.

Department of Mathematics

KAIST

373-1 Kusong-dong, Yusong-ku

Taejon 305-701

Korea

e-mail: khkwon@jacobi.kaist.ac.kr 